\title{
Retracted Article: Rational Curves and Prolongations of $G$-structures
}

\author{
Jun-Muk HWANG \\ Korea Institute for Advanced Study, Hoegiro 85, Seoul 02455, Korea \\ E-mail: jmhwang@kias.re.kr
}

The Author has retracted the article. After publication, it was pointed out to the author that there is a serious gap in the proof of the main result. The online version of this article contains the full text of the retracted article as electronic supplementary material. 